\title{
O TEMPO E A DISTÂNCIA: AVALIAÇÃO ON-LINE DAS HABILI- DADES DE ADMINISTRAÇÃO DO TEMPO
}

\author{
TIME AND DISTANCE: evaluation on-line of time management skills \\ EL TIEMPO Y LA DISTANCIA: evaluación online de las habilidades de adminis- \\ tración del tiempo.
}

\author{
Euro Marques Júnior \\ Professor Doutor da Faculdade de Agudos (FAAG). \\ euro.marques@faag.com.br \\ José Dutra de Oliveira Neto \\ Professor Doutor da Faculdade de Economia, Administração \\ e Contabilidade de Ribeirão Preto (FEA-RP/USP). \\ dutra@usp.br \\ Emília de Mendonça Rosa Marques \\ Professora Doutora da Universidade Estadual Paulista (UNESP). \\ emilia@fc.unesp.br \\ Robson Delfino Rosano \\ Supervisor do INEPAD / Mestre. \\ robson@inepad.org.br
}

RESUMO: A administração do tempo é tema relevante para a sociedade moderna, sendo foco de estudos específicos voltados para a produtividade industrial, sucesso profissional, realização pessoal e desempenho acadêmico. A Associação Brasileira de Educação a Distância aponta como um dos principais motivos dados pelos alunos para a evasão a "falta de tempo para estudar", tornando, portanto, necessário aprofundar o estudo das habilidades de administração do tempo com o objetivo de superar essa barreira ao ensino por meio da EAD (Educação a Distância) através da Internet. Dessa forma, este trabalho caracteriza as habilidades de gerenciamento do tempo e apresenta um instrumento de pesquisa on-line capaz de mensurá-las. Como demonstração de sua aplicação, utiliza-se o instrumento em cinco amostras de estudantes de cursos mistos ou on-line, avaliando 505 respondentes. Os resultados apontam, por um lado, que as habilidades de administração do tempo podem se aprimorar com o amadurecimento do estudante, independentemente de seu gênero. Por outro lado, a formação acadêmica, por si só, não leva a um aumento significativo das habilidades de gerenciamento do tempo dos estudantes. $O$ instrumento apresentado se diferencia por ser voltado para a avaliação de estudantes de cursos EAD, podendo ser respondido on-line de forma rápida por possuir apenas cinco itens. $O$ instrumento mensura as habilidades de gestão do tempo do estudante e da turma para que os responsáveis pelo curso possam tomar medidas no sentido de adaptar os recursos do Ambiente Virtual de Aprendizagem ao comportamento da turma e oferecer treinamentos para desenvolver as habilidades dos alunos nessa área.

PALAVRAS-CHAVE: Administração do tempo. Educação a distância. Ambiente Virtual de Aprendizagem.

ABSTRACT: Time management is an important issue for modern society and also is focus specific studies focused in industrial productivity, professional success, personal achievement and academic performance. The Brazilian Association of Distance Education points out one of the main reasons given by students for evasion as "lack of time to study." Therefore, it is necessary to deepen the study of time management skills, to overcome this barrier to education through ODL (open and distance learning) in the Internet. Thus, this article characterizes the time management skills and presents an online research tool capable of to measure them. As a demonstration application, the instrument is used in five samples of mixed courses or online, evaluating 505 respondents. The results show, on the one hand, that the time management skills can improve with the student's maturity, regardless of their gender. On the other hand, academic education alone does not lead to a significant increase in students' time management skills. The described instrument is distinguished by being focused on the evaluation of students in distance education courses that can be completed on-line quickly, as it has only five items. The instrument measures the student's time management skills and the class so that course responsibles take steps to adapt the resources of the Virtual Learning Environment to the class behavior and provide training to develop students' skills in this area.

KEYWORDS: Time Management. Distance education. Virtual learning environment.

$\overline{\text { Artigo recebido em maio de } 2015}$

Aprovado em julho de 2015

Cad. Pes., São Luís, v. 22, n. 2, mai./ago. 2015 
RESUMEN: La administración del tiempo es un tema importante para la sociedad moderna, con estudios específicos centrados en la productividad industrial, el éxito profesional, la realización personal y el rendimiento académico. La Asociación Brasileña de Educación a Distancia señala una de las principales razones dadas por los estudiantes para la evasión como la "falta de tiempo para estudiar", así, es necesario profundizar el estudio de las habilidades de gestión del tiempo, con el objetivo de superar esta barrera a la enseñanza por medio de la EAD (Educación a Distancia) a través de la Internet. Por lo tanto, este artículo caracteriza las habilidades de gestión del tiempo y presenta un instrumento de investigación capaz de medirlas. Como demonstración de su aplicación, se utiliza el instrumento en cinco muestras de estudiantes en cursos mixtos o online, mediante la evaluación de 505 encuestados. Los resultados muestran, por un lado, que las habilidades de gestión del tiempo pueden mejorar con la madurez del alumno, independientemente de su género. Por otro lado, la formación académica por sí sola no lleva a un aumento significativo de las habilidades de gestión del tiempo de los estudiantes. El instrumento presentado se distingue por regresar para la evaluación de los estudiantes en cursos EAD, se puede completar online rápidamente al tener sólo cinco artículos. El instrumento mide las habilidades de gestión del tiempo del estudiante y de la clase para que los responsables por lo curso puedan tomar medidas para adaptar los recursos del Ambiente Virtual de Aprendizaje para el comportamiento de la clase y ofrecer capacitación para desarrollar habilidades de los estudiantes en esta área.

PALABRAS CLAVE: Gestión del tiempo. Educación a Distancia. Ambiente Virtual de Aprendizaje. 
O TEMPO E A DISTÂNCIA | Euro Marques Júnior et. al.

\section{1 | INTRODUÇÃO}

A Educação a Distância (EAD) através da Internet é um novo paradigma que abre muitas possibilidades para o uso de novas tecnologias de informação e comunicação que contribuam com o ensino e treinamento de pessoas que teriam dificuldade em acessar esse conteúdo de forma exclusivamente presencial, seja pela distância à escola ou pela dificuldade de conciliar os horários de estudo e trabalho.

O design instrucional é o campo do conhecimento que busca aprimorar o planejamento e a aplicação dos métodos, visando facilitar os processos de ensino-aprendizagem, sendo de grande importância para a EAD. Já o design de interação é uma área do design especializada no projeto de artefatos interativos como websites, jogos eletrônicos e softwares. O foco do design de interação são as relações humanas tecidas por meio de artefatos interativos que funcionam também como meios de comunicação interpessoal. O design instrucional e o design de interação podem ajudar a desenvolver as características do Ambiente Virtual de Aprendizagem (AVA) para facilitar a aprendizagem colaborativa assistida por computadores (Computer Supported Collaborative Learning - CSCL).

Diversos autores como Trueman e Hartley, Ostlund, Hargittai, Livingstone e Helsper, entre outros, apontam a influência da idade, sexo, nível de escolaridade e motivação do estudante sobre vários aspectos dos cursos de EAD, desde o desempenho na aprendizagem até a participação nas atividades e evasão dos cursos. Esses aspectos são importantes, mas de modo geral, os responsáveis pelos cursos de EAD não têm como controlá-los, pois recebem os alunos na situação em que se encontram e apenas os incluem no curso já estruturado.

Contudo, existem outras características individuais que podem ser mensuradas antes do início do curso EAD e aprimoradas por meio de treinamentos e adaptações nos recursos do Ambiente Virtual de Aprendizagem para que os estudantes possam ter uma melhor experiência de aprendizagem on-line.

A revisão da literatura aponta a gestão do tempo como uma dessas características que pode ser desenvolvida e pode influenciar nos resultados de cursos EAD. As habilidades de gestão do tempo abrangem a capacidade de definir metas, priorizar, planejar e programar atividades. Com essas habilidades bem desenvolvidas os estudantes tornar-se-ão mais eficientes e organizados, facilitando sua participação nos cursos EAD. Por exemplo, de acordo com o INEP (Instituto Nacional de Estudos e Pesquisas Educacionais Anísio Teixeira), a evasão nos cursos de graduação na modalidade a distância foi de $17 \%$ de 2011 para 2012, e de 30\%, comparandose 2012 a 2013 (BRASIL, 2015). O CensoEAD.Br, realizado pela ABED (Associação Brasileira de Educação a Distância), aponta os principais motivos dados pelos alunos para a evasão como sendo "falta de tempo para estudar" e "falta de adaptação" à rigorosa metodologia dos cursos EAD. Portanto, torna-se necessário aprofundar o estudo das habilidades de administração do tempo para superar essa barreira no ensino, através da EAD.

Dessa forma, o objetivo deste trabalho é caracterizar as habilidades de gerenciamento do tempo e apresentar um instrumento de pesquisa on-line capaz de mensurá-las. Como demonstração de aplicação, o instrumento é utilizado em cinco amostras diferentes que avaliam 505 respondentes. 
O TEMPO E A DISTÂNCIA | Euro Marques Júnior et. al.

\section{2 | GESTÃO DO TEMPO}

A administração do tempo é um tema relevante para a sociedade moderna, tendo estudos específicos voltados para a produtividade industrial, realização profissional, sucesso pessoal e desempenho acadêmico. Race (1992), por exemplo, afirma que ter habilidades bem desenvolvidas de gerenciamento do tempo é a chave para ser bem sucedido nos estudos, e é fundamental para evitar todos os tipos de problemas na vida acadêmica. Se isso é verdade para a educação tradicional, deve ser muito mais crítico para a EAD, em que o aluno virtual precisa gerenciar de forma autônoma suas atividades e tarefas on-line, convivendo com agendas e cronogramas diversos sem abrir mão de outras atividades do ambiente "real".

De acordo com Barbour e Reeves (2009), os benefícios associados com a escolaridade virtual são a expansão do acesso educacional, oferecendo oportunidades de aprendizagem de alta qualidade, capazes de melhorar os resultados alcançados e as competências dos alunos. A EAD também permite uma maior liberdade de escolha educacional, uma vez que possui menores restrições quanto ao horário e ao local onde acontece a aprendizagem. Desta forma é possível alcançar uma maior eficiência administrativa no uso dos recursos públicos e privados usados na educação. No entanto, a investigação para apoiar essas conjecturas ainda é limitada. Os desafios associados com a escolaridade virtual incluem a conclusão de que os únicos estudantes tipicamente bem sucedidos em ambientes de aprendizagem on-line são aqueles que têm orientações independentes em relação à aprendizagem, são altamente motivados por fontes intrínsecas e apresentam fortes habilidades de gerenciamento do tempo e de uso da tecnologia. Estas características são tipicamente associadas com os alunos adultos. O problema com esse enfoque é que os adultos aprendem de forma diferente dos estudantes mais jovens. Necessita-se de mais investigação sobre os fatores que explicam o sucesso do aluno adolescente na educação a distância e mais pesquisas que possam ir além das habituais comparações de desempenho dos alunos nas escolas tradicionais e virtuais.

Trueman e Hartley (1996) apresentam uma escala de mensuração da habilidade de gestão do tempo com 14 itens, aplicando-a a um grupo de estudantes. Os resultados indicaram que as mulheres, em geral, relataram significativamente maiores habilidades de gerenciamento de tempo do que os homens. Já os estudantes com mais idade relataram significativamente melhores habilidades de gerenciamento de tempo do que os mais jovens. O desempenho acadêmico, no entanto, foi apenas modestamente relacionado à idade e aos componentes da escala de gerenciamento de tempo.

O estudo de Lahmers e Zulauf (2000) revelou que as habilidades de gestão do tempo e a contagem do tempo dedicado aos estudos estavam positivamente associados aos resultados do Grade Average Point (GPA), uma medida de desempenho acadêmico.

Alguns estudos mostram as diferenças existentes entre as percepções dos estudantes, dependendo de sua formação e experiência anterior. Os estudantes de graduação, por exemplo, salientaram os aspectos sociais e práticos do curso, enquanto os estudantes de mestrado, em maior medida, enfatizaram os aspectos cognitivos. A quantidade de interações espontâneas também foi maior no grupo de graduação: esses estudantes precisaram de mais apoio dos outros, a fim de gerir os seus estudos em comparação com os estudantes de mestrado (OSTLUND, 2008).

Autores como Macan et al. (1990) e Britton e Tesser (1991) têm estudado a capacidade de gestão do tempo de estudantes universitários, analisando as correlações entre o desempenho acadêmico e estresse, e efeitos de práticas de gestão de tempo sobre as notas.

Já autores como Senecal, Koestner e Vallerand (1995), Chu e Choi (2005), Lay e Schouwenburg (1993) e Michinov et al. (2011), têm estudado a procrastinação acadêmica. Senecal, Koestner 
O TEMPO E A DISTÂNCIA | Euro Marques Júnior et. al.

e Vallerand (1995) avaliaram o papel da autorregulação como um preditor da procrastinação acadêmica. Estudantes franco-canadenses de uma faculdade $(\mathrm{N}=498)$ completaram a Escala de Motivação Acadêmica, bem como uma Escala de Procrastinação Acadêmica e outras medidas (ansiedade, autoestima e depressão) que estavam relacionadas com o medo do fracasso. Os resultados da análise de correlação indicaram que os alunos com motivos intrínsecos para completar tarefas acadêmicas procrastinaram menos do que aqueles com menos razões autônomas. Resultados da análise de regressão indicaram que as medidas de depressão, autoestima e ansiedade são responsáveis por $14 \%$ da variação na procrastinação acadêmica, enquanto as variáveis de autorregulação, respondem por $25 \%$. Estes resultados apoiam a noção de que a procrastinação é um problema motivacional que envolve mais do que a falta de habilidades de gerenciamento de tempo ou preguiça.

Segundo Lay e Schouwenburg (1993), os alunos procrastinadores obtiveram escores mais baixos em três subescalas de gerenciamento de tempo em comparação com os não-procrastinadores, e relataram níveis mais elevados de comportamentos dilatórios. As subescalas de gerenciamento de tempo estavam negativamente relacionadas com o comportamento dilatório em algumas das tarefas do curso. A procrastinação estava positivamente relacionada com o desânimo no início do curso antes de fazer planos de estudo e trabalho e positivamente relacionada com a agitação (nervosismo) vivida durante a última semana de aula. A subescala de gerenciamento de tempo, percepção de controle do tempo, estava negativamente relacionada com a agitação em cada avaliação. Mais importante, a baixa percepção de controle do tempo associada com os alunos procrastinadores parece mediar os seus níveis mais elevados de agitação durante a última semana de aula.

Ainda nessa área, Michinov et al. (2011) realizaram um estudo centrado na procrastinação e seu papel em um ambiente de aprendizagem online. Esperava-se que a procrastinação iria influenciar o êxito da aprendizagem on-line e que isso poderia ser explicado pelo nível de participação dos alunos em fóruns de discussão. O estudo foi realizado para testar esta hipótese em uma amostra de estudantes que faziam um curso de 10 semanas sobre questões ambientais e de uso da terra. Como previsto, foi encontrada uma relação negativa entre a procrastinação e o desempenho, e essa relação foi mediada pelo nível de participação dos estudantes em fóruns de discussão. Em outras palavras, parece que se os maiores procrastinadores são alunos on-line menos bem sucedidos do que os menos procrastinadores, isso se deve em parte devido à falta de participação, desses alunos, em fóruns de discussão durante o processo de aprendizagem.

Prichard, Stratford e Bizo (2006) sugerem que o treinamento para trabalhar em equipe estimula os integrantes do grupo a exercerem estratégias de gestão do tempo e igualdade de participação, que, por sua vez, aumentam a aprendizagem por parte de cada aluno.

De acordo com Yedidia et al. (2003), o ensino de habilidades de comunicação melhorou significativamente a competência de comunicação global dos alunos de medicina, as suas competências na construção de relacionamentos, na organização e gestão do tempo, na avaliação do paciente, bem como nas tarefas de negociação e decisão compartilhada que são importantes para se alcançar resultados positivos com os pacientes.

Já Macan (1994) tem uma posição mais cética em relação aos efeitos do treinamento em gestão do tempo. Para ele, apesar da literatura popular na gestão do tempo afirmar que o envolvimento em comportamentos de gerenciamento de tempo resulta em maior desempenho e satisfação no trabalho e menos tensões, ainda falta uma estrutura teórica e exames empíricos para confirmar tais suposições. O autor propôs e testou um modelo de processo de gestão do tempo por meio da aplicação de diversas escalas com empregados e avaliações de desempenho feitas pelos supervisores. O exame dos coeficientes do modelo sugere que se envolver em alguns comportamentos 
de gerenciamento de tempo pode trazer efeitos benéficos sobre as tensões e satisfação no trabalho, mas não sobre o desempenho nesse ambiente. Contrariamente às afirmações populares, o treinamento em gestão do tempo nem sempre é eficaz, uma vez que o desempenho depende de outros fatores, como motivação e outras habilidades.

Solimeno et al. (2008) compararam a eficácia da aprendizagem face a face com a da aprendizagem colaborativa assistida por computador (CSCL) no aumento do conhecimento acadêmico e das competências profissionais. Também exploraram como as características de personalidade e estratégias de aprendizagem dos alunos foram associadas com melhores resultados de aprendizagem em contextos on-line ou face a face. Cento e setenta estudantes participaram de 10 seminários de psicologia, cinco on-line e cinco face a face. O aprendizado acadêmico e profissional aumentou para os participantes em ambas as configurações. Os resultados globais mostram que a aprendizagem colaborativa on-line assíncrona pode aumentar as competências profissionais normalmente aprendidas apenas em contextos educativos face a face, e que a CSCL pode ser usada para fornecer oportunidades educacionais inovadoras que se adaptam às necessidades particulares de alunos com baixa ansiedade, alta eficácia em resolver problemas, mas que tinham problemas de gerenciamento de tempo em suas estratégias de aprendizagem.

Portanto, fica evidente a necessidade de se avaliar as habilidades de gestão do tempo dos estudantes para que os responsáveis pelos cursos EAD possam adequar as atividades on-line e cronogramas propostos às necessidades da turma, oferecendo apoio para lembrar as datas críticas e acompanhar o desempenho nas tarefas.

Esses aspectos levantados permitem elaborar a hipótese de que alunos com maior nível de capacidade de gestão do tempo terão uma maior facilidade para desempenhar suas atividades no Ambiente Virtual de Aprendizagem.

\section{3 | METODOLOGIA}

Utilizou-se a metodologia de pesquisa survey, em sua modalidade on-line, como instrumento de pesquisa. A análise proposta é quantitativa, a qual se deu sob uma perspectiva sistêmica e multidimensional, confrontando os resultados obtidos com o referencial teórico utilizado.

Para a obtenção dos dados desta pesquisa aplicou-se questionários via internet (Web-Survey), divulgados na plataforma Moodle e disponibilizados no site Survey Monkey (www.surveymonkey. com). Os resultados dos dados coletados no questionário com questões fechadas foram postos em gráficos, facilitando sua análise e descrição.

O questionário utilizado segue uma estrutura matricial de respostas com uma escala tipo Likert de cinco pontos com os extremos "discordo totalmente" e "concordo totalmente".

Esse questionário é parte integrante do instrumento de pesquisa para mensurar as habilidades de gestão do uso do tempo, o nível de extroversão e de habilidades sociais dos alunos que foi desenvolvido em 2011, em sua primeira versão (TES 1.0), com 57 itens ligados à extroversão, 12 itens ligados à gestão do tempo e 11 itens relacionados às habilidades sociais. Os 80 itens foram criados a partir da literatura da área e adaptados para o público alvo formado por alunos de cursos EAD, jovens e adultos. Após uma análise estatística multivariada dos resultados obtidos, foi possível uma alteração do TES 1.0, reduzindo-se o número de itens e modificando-se os itens ligados à gestão do tempo da lógica inversa para a lógica direta. Utilizou-se uma análise dos componentes principais, mantendo-se os cinco itens mais representativos de cada tema do questionário. 
O TEMPO E A DISTÂNCIA | Euro Marques Júnior et. al.

\subsection{Questionário de avaliação de uso do tempo}

Este artigo apresenta o componente de gestão do tempo da segunda versão do instrumento (TES 2.0), em que nenhuma das cinco questões foi elaborada com lógica inversa, sendo assim o valor 5 (cinco) representa sempre o maior nível de atendimento à característica ou prática que está sendo avaliada, na opinião do respondente.

O coeficiente Alfa de Cronbach é uma forma de estimar a confiabilidade de um questionário aplicado em uma pesquisa, pois mede a correlação entre respostas em um questionário por meio da análise das respostas dadas pelos respondentes, apresentando uma correlação média entre as perguntas, podendo variar de 0 a 1.

A tabela 1 apresenta o Alfa de Cronbach calculado com o software IBM SPSS para o componente ligado à Gestão do Tempo. Como o valor de alfa está entre 0,7 e 0,8, podemos considerar que o instrumento possui uma confiabilidade aceitável (GEORGE \& MALLERY, 2002).

Tabela 1 - Coeficiente Alfa de Cronbach

\begin{tabular}{|ccc|}
\hline Componente & Alfa de Cronback & Tamanho da Amostra \\
\hline Gestão do Tempo & 0,733 & 505 \\
\hline Fonte: Elaborado pelos autores. & &
\end{tabular}

Os cinco itens servem de base para o cálculo do nível de habilidades de gestão do tempo da amostra, calculados pela média das respostas. O grau de proficiência será avaliado por uma escala exponencial, refletindo e destacando a importância da quantidade de respostas que possuam o nível mais alto de concordância com as questões, conforme o quadro 1.

Quadro 1 - Escala de avaliação da proficiência

\begin{tabular}{|cc|}
\hline Grau de Proficiência & Avaliação \\
\hline até 3,5 & Baixo \\
3,6 a 4,2 & Moderado \\
4,3 a 4,6 & Alto \\
4,7 a 5 & Muito alto \\
\hline
\end{tabular}

Fonte: Elaborado pelos autores.

A variável Gestão do Tempo pode ser compreendida como "habilidade em gerenciar o tempo", e é definida pela média das cinco questões ligadas ao tema, conforme o quadro 2.

Quadro 2 - Itens componentes da variável Gestão do Tempo.

\begin{tabular}{|l|}
\multicolumn{1}{c|}{ Gestão do Tempo } \\
\hline 1. Geralmente planejo as atividades do meu próximo dia de trabalho. \\
2. Poucas vezes deixo sem terminar o que começo. \\
3. O planejamento ajuda a resolver o meu problema de falta de tempo. \\
4. Minhas gavetas estão sempre em ordem. \\
5. Prefiro planejar com antecedência do que resolver as coisas na hora. \\
\hline
\end{tabular}

Fonte: Elaborado pelos autores. 
O TEMPO E A DISTÂNCIA | Euro Marques Júnior et. al.

Dessa forma, espera-se que um estudante que tenha o valor um (1) em Gestão do Tempo apresente grande dificuldade em gerenciar o tempo, procrastinando e entregando suas atividades com atrasos, enquanto um estudante que tenha o valor cinco (5) em Gestão do Tempo apresente grande capacidade de gerenciar seu tempo, completando suas atividades dentro dos prazos propostos.

\subsection{Descrição das amostras}

Colaboraram com esta pesquisa 663 respondentes, consultados em um período de junho de 2011 a julho de 2014. As amostras podem ser agrupadas de acordo com o curso que os respondentes faziam no momento da pesquisa. Percebem-se variações na idade, nível de escolaridade e distribuição por sexo em cada grupo, evidenciando a heterogeneidade da amostra, conforme as figuras 1 e 2 .

Como os questionários foram respondidos voluntariamente não foi feita uma análise dos não respondentes. Os casos foram analisados individualmente, removendo-se aqueles que apresentaram dados incompletos.

Figura 1 - Distribuição quantitativa por sexo em cada amostra

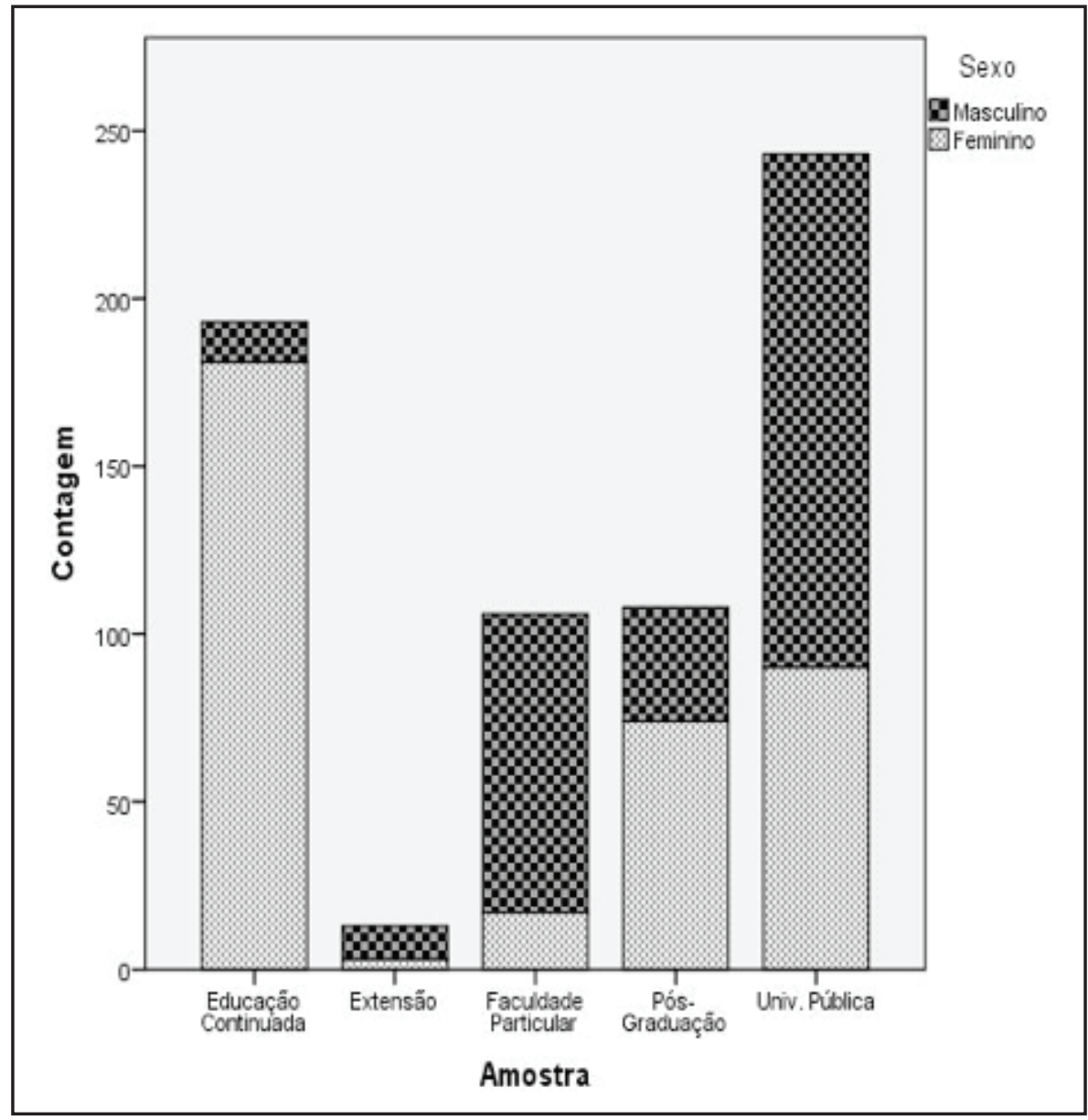

Fonte: Elaborado pelos autores. 
O TEMPO E A DISTÂNCIA | Euro Marques Júnior et. al.

Figura 2 - Distribuição percentual por escolaridade em cada amostra

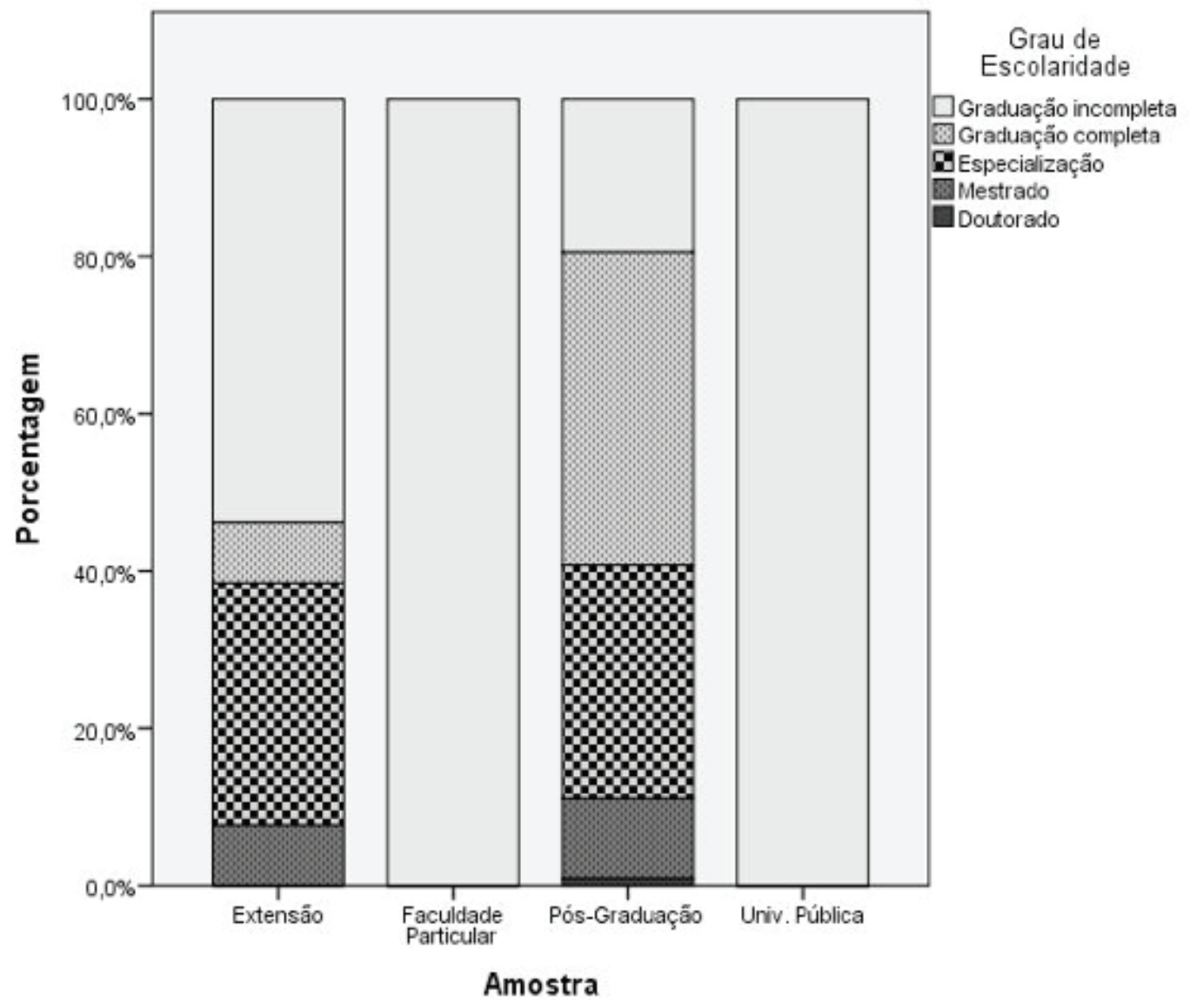

Fonte: Elaborado pelos autores.

O levantamento de dados foi feito entrevistando-se alunos que participavam de disciplinas ou cursos ministrados pelos autores que voluntariamente responderam aos questionários on-line. Vale ressaltar que, apesar de não ser uma amostragem aleatória, ela atende inteiramente os objetivos desta pesquisa.

A seguir, será apresentada uma descrição geral dos respondentes, agrupados de acordo com o curso que estavam fazendo no momento da pesquisa.

\subsubsection{Estudantes de Universidade Pública}

A amostra se constituiu de 243 alunos de uma universidade pública do estado de São Paulo (Brasil), cursando disciplinas presenciais na área de Economia e Matemática, usando a Plataforma Moodle como suporte didático, que, voluntariamente, responderam aos questionários de 2012 a 2014.

\subsubsection{Estudantes de Faculdade Particular}

A amostra, coletada em 2013, foi formada por 106 estudantes de uma faculdade particular do estado de São Paulo, com uma média de idade de 33 anos, sendo $91 \%$ do sexo masculino e $9 \%$ do sexo feminino. Trata-se de um curso misto (blended), pois a disciplina presencial, na área de Engenharia, usou a Plataforma Moodle como suporte didático. Além de repositório do material didático, o Moodle foi usado para atividades on-line e fóruns de discussão. 
O TEMPO E A DISTÂNCIA | Euro Marques Júnior et. al.

\subsubsection{Estudantes de Educação Continuada}

A amostra, coletada em 2011, contou com 196 professores do ensino fundamental do estado de São Paulo, da área de Matemática e de Alfabetização/Linguagem. Quanto ao perfil, obteve-se que 93\% dos respondentes eram do sexo feminino, com uma média de idade em torno de 41 anos; 99\% possuíam graduação, 10\% estavam vinculados a uma pós-graduação e $48 \%$ já a haviam concluído.

\subsubsection{Estudantes de Curso de Extensão}

A amostra, coletada em 2014, se constituiu de 13 alunos de um curso de extensão na área de estatística oferecido por uma universidade pública do estado de São Paulo. Desses, 54\% eram do sexo masculino, com uma média de idade de 32 anos e $46 \%$ do sexo feminino, com uma média de idade de 29 anos. Outro dado aponta que $7 \%$ ainda estavam cursando a graduação e $37 \%$ já haviam se formado, mas não possuíam outros cursos. $12 \%$ já tinham especialização, 35\% já possuíam mestrado e $9 \%$ doutorado. O curso foi desenvolvido completamente a distância, no período de 6 de janeiro a 7 de fevereiro de 2014, com uso de materiais audiovisuais, fóruns de discussão e apoio de um tutor on-line utilizando a Plataforma Moodle.

\subsubsection{Estudantes de Pós-Graduação}

A amostra, coletada em 2013 e 2014, foi composta por 108 alunos, no Brasil, participando de cursos de pós-graduação misto (blended) que usaram a Plataforma Moodle como suporte didáti$\mathrm{co}$, que, voluntariamente, responderam aos questionários. A média de idade foi de 41 anos, $68 \%$ eram do sexo feminino e $32 \%$ eram do sexo masculino.

\section{4 | RESULTADOS}

A variável Gestão do Tempo, definida como "nível de habilidade do respondente em gerenciar o tempo", é determinada pela média das cinco questões ligadas ao tema, conforme o quadro 2. Desta forma, o instrumento on-line apresentado neste artigo aparenta ser mais rápido de se preencher e de se analisar do que o proposto por outros autores como Trueman e Hartley (1996) que propuseram um instrumento com 14 itens.

A tabela 2 apresenta a média e o desvio padrão para esta variável, considerando todos os respondentes.

Tabela 2 - Estatísticas descritivas da variável Gestão do Tempo

\begin{tabular}{|lccc|}
\hline & N & Média & Desvio padrão \\
\hline Gestão do Tempo & 505 & 3,9493 & 0,70753 \\
\hline Fonte: Elaborado pelos autores. & & &
\end{tabular}

De modo geral, concordando com Race (1992) e Lahmers e Zulauf (2000), percebe-se a necessidade do oferecimento de treinamento para que os estudantes possam desenvolver suas habilidades de gerenciamento do tempo e ter um melhor desempenho nos estudos on-line.

A figura 3 apresenta um gráfico que compara as médias da habilidade de gestão do tempo das amostras. Percebe-se que apenas a amostra composta por estudantes do curso de Educação Continuada está com um alto grau de habilidades de administração do tempo, estando as demais com um grau moderado. 
O TEMPO E A DISTÂNCIA | Euro Marques Júnior et. al.

Como indicado por Michinov et al. (2011), esta deficiência no nível de habilidades de gestão do tempo pode acarretar em uma série de problemas para o desenvolvimento individual do aluno, bem como pode comprometer o cronograma do curso, que, muitas vezes, precisa adiar prazos e reabrir a oportunidade para que alunos atrasados possam completar as atividades.

Apesar de Senecal, Koestner e Vallerand (1995) apontarem que a procrastinação é um problema motivacional que envolve mais do que a falta de habilidades de gerenciamento de tempo ou preguiça, Lay e Schouwenburg (1993) indicam que o nível das habilidades de gerenciamento do tempo está negativamente relacionado com o comportamento dilatório. O instrumento proposto pode apoiar, portanto, a avaliação do comportamento das turmas em relação à procrastinação.

Figura 3 - Média da Gestão do Tempo por cada amostra

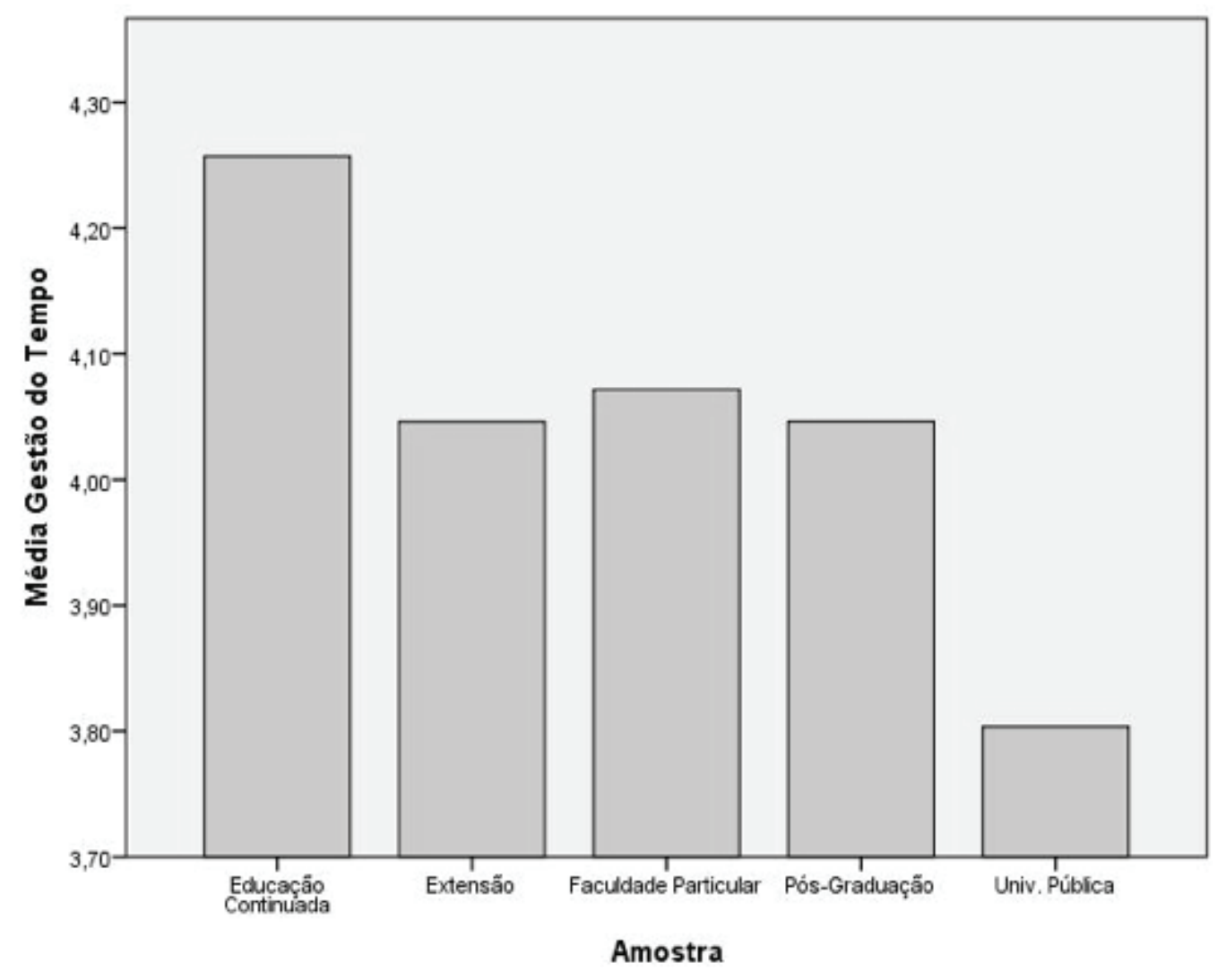

Fonte: Elaborado pelos autores.

A tabela 3 apresenta as correlações entre as variáveis. Os resultados deste trabalho concordam parcialmente com os de Trueman e Hartley (1996), uma vez que os estudantes com maior idade apresentaram significativamente melhores habilidades de gerenciamento de tempo do que os mais jovens. Contudo, as mulheres, em geral, não relataram significativamente maiores habilidades de gerenciamento de tempo do que os homens, neste estudo. Ou seja, não houve correlação do gênero com a gestão do tempo na amostra estudada.

Do mesmo modo, os estudantes com maior nível de escolaridade não relataram significativamente maiores habilidades de gerenciamento de tempo do que os com menor escolaridade, apesar da escolaridade estar positivamente correlacionada com a idade. 
O TEMPO E A DISTÂNCIA | Euro Marques Júnior et. al.

Tabela 3 - Correlações entre as variáveis

\begin{tabular}{|c|c|c|c|c|}
\hline & & Idade & Escolaridade & Sexo \\
\hline \multirow{3}{*}{$\begin{array}{l}\text { Gestão do } \\
\text { Tempo }\end{array}$} & Correlação de Pearson & $0,183^{* *}$ & 0,089 & 0,058 \\
\hline & Sig. (2 extremidades) & 0,000 & 0,054 & 0,190 \\
\hline & $\mathrm{N}$ & 501 & 470 & 505 \\
\hline \multirow{3}{*}{ Idade } & Correlação de Pearson & 1 & $0,573^{* *}$ & $0,157^{* *}$ \\
\hline & Sig. (2 extremidades) & & 0,000 & 0,000 \\
\hline & $\mathrm{N}$ & 501 & 466 & 501 \\
\hline \multirow{3}{*}{$\begin{array}{l}\text { Grau de } \\
\text { Escolaridade }\end{array}$} & Correlação de Pearson & $0,573^{* *}$ & 1 & $0,257^{* *}$ \\
\hline & Sig. (2 extremidades) & 0,000 & & 0,000 \\
\hline & $\mathrm{N}$ & 466 & 470 & 470 \\
\hline
\end{tabular}

Fonte: Elaborado pelos autores.

Por um lado, os resultados apontam que as habilidades de administração do tempo podem se aprimorar com o amadurecimento do estudante, independente de seu sexo. Por outro lado, a formação acadêmica, por si só, não levou a um aumento significativo das habilidades de gerenciamento do tempo dos estudantes da amostra.

Como Prichard, Stratford e Bizo (2006) e Yedidia et al. (2003) sugerem que o treinamento para trabalhar em equipe e o ensino de habilidades de comunicação podem melhorar significativamente as habilidades de gestão do tempo dos estudantes, os resultados apontam a necessidade de mais estudos que apoiem o desenvolvimento de treinamentos nessa área.

\section{5 | CONSIDERAÇÕES FINAIS}

Existem diversos instrumentos de mensuração de habilidades de administração do tempo criados com propósitos e enfoques específicos. O instrumento apresentado se diferencia por ser voltado para a avaliação de estudantes de cursos EAD, podendo ser respondido on-line de forma rápida, pois possui apenas cinco itens. $O$ instrumento mensura as habilidades de gestão do tempo do estudante e da turma para que os responsáveis pelo curso possam tomar medidas no sentido de adaptar os recursos do AVA ao comportamento da turma e de oferecer treinamentos para desenvolver as habilidades dos alunos nesta área.

Os resultados apresentados apontam que as habilidades de gerenciamento do tempo tendem a se aprimorar com o amadurecimento do estudante, independentemente de seu gênero. Segundo Barbour e Reeves (2009), para que os estudantes do Ensino Médio sejam bem sucedidos em ambientes de aprendizagem on-line eles devem possuir fortes habilidades de gerenciamento do tempo. Desta forma, em trabalhos futuros pretende-se a aplicação do instrumento proposto para avaliar estudantes em nível de escolaridade e idade anteriores à graduação. Trata-se de uma proposta factível, uma vez que o instrumento é adequado também a esses estudantes e pelo fato de que mais pesquisas nesse nível de escolaridade são necessárias, visando melhor compreensão desse público e maior adequação nas propostas de cursos. 
O TEMPO E A DISTÂNCIA | Euro Marques Júnior et. al.

Sabe-se que a gestão do tempo, além dos aspectos motivacionais, é parte importante numa característica dos estudantes, a procrastinação. Conforme o estudo de alguns pesquisadores (MICHINOV et al., 2011), a procrastinação influencia no êxito da aprendizagem on-line. A formação acadêmica, por si só, não leva a um aumento significativo das habilidades de gerenciamento do tempo dos estudantes. Desta forma observa-se a necessidade de propostas educacionais que apresentem ambientes e recursos preparados para auxiliar os estudantes no desenvolvimento dessa habilidade, principalmente por meio de uma estruturação adequada das atividades (SOLIMENO et al., 2008).

Neste estudo não se buscou verificar a relação entre o nível de habilidade de gestão do tempo e a pontualidade na realização das tarefas ou o desempenho acadêmico. Espera-se verificar esses aspectos em estudos futuros, sugerindo-se também uma comparação entre este instrumento de pesquisa e outras escalas de mensuração de habilidades de administração do tempo.

\section{Agradecimentos}

Agradecimentos à CAPES (23038.032613/2009-21) e ao INEPAD pela ajuda financeira e o apoio na realização da pesquisa. 
O TEMPO E A DISTÂNCIA | Euro Marques Júnior et. al.

\section{Referências}

BARBOUR, M. K.; REEVES, T. C. The reality of virtual schools: a review of the literature. Computers \& Education, v. 52, n. 2, p. 402-416, Feb. 2009. ISSN 03601315. Disponível em: <http://www.sciencedirect.com/ science/article/pii/S0360131508001450 >. Acesso em: 10 jan. 2015.

BRITTON, B. K.; TESSER, A. Effects of time-management practices on college grades. Journal of Educational Psychology, v. 83, n. 3, p. 405-410, Sept. 1991. ISSN 0022-0663. Disponível em: <http://psycnet.apa. org/psycinfo/1992-10822-001>. Acesso em: 10 jan. 2015.

$\mathrm{CHU}$, A. H. C.; $\mathrm{CHOI}$, J. N. Rethinking procrastination: positive effects of "active" procrastination behavior on attitudes and performance. Journal of Social Psychology, v. 145, n. 3, p. 245-264, June 2005. ISSN $0022-$ 4545. Disponível em: <http://www.ncbi.nlm.nih.gov/ pubmed/15959999>. Acesso em: 10 jan. 2015.

GEORGE, D.; MALLERY, P. SPSS for windows step by step: a simple guide and reference, 11.0 update. 4. ed. Boston: [s.n.], 2002.

INSTITUTO NACIONAL DE ESTUDOS E PESQUISAS EDUCACIONAIS ANÍSIO TEIXEIRA. Censo da Educação Superior. Brasília, DF, 2015. Disponível em: <http://portal.inep.gov.br/web/censo-da-educacao-superior >. Acesso em: $10 \mathrm{dez} .2015$.

LAHMERS, A. G.; ZULAUF, C. R. Factors associated with academic time use and academic performance of college students: a recursive approach. Journal of College Student Development, US, v. 41, n. 5, p. 544-556, 2000. ISSN 0897-5264.

LAY, C. H.; SCHOUWENBURG, H. C. Trait procrastination, time management, and academic behavior. Journal of Social Behavior and Personality, v. 8, n. 4, p. 647-662, 1993. ISSN 0886-1641. Disponível em: < http://psycnet.apa.org/psycinfo/1994-23368-001>. Acesso em: 10 jan. 2015.

MACAN, T. H. et al. College students time management: correlations with academic performance and stress. Journal of Educational Psychology, v. 82, n. 4, p. 760-768, Dec. 1990. ISSN 0022-0663. Disponível em: <http://psycnet.apa.org/journals/edu/82/4/760.html>. Acesso em: 10 jan. 2015.

MACAN, T. H. Time management: test of a process model. Journal of Applied Psychology, v. 79, n. 3, p. 381-391, June 1994. ISSN 0021-9010. Disponível em: <http://psycnet.apa.org/journals/apl/79/3/381>. Acesso em: 10 jan. 2015.
MICHINOV, N. et al. Procrastination, participation, and performance in online learning environments. Computers \& Education, v. 56, n. 1, p. 243-252, Jan. 2011. ISSN 0360-1315. Disponível em: <http://www.sciencedirect.com/science/article/pii/S0360131510002204>. Acesso em: 10 jan. 2015.

OSTLUND, B. Prerequisites for interactive learning in distance education: perspectives from swedish students. Australasian Journal of Educational Technology, v. 24, n. 1, p. 42-56, 2008. ISSN 1449-3098. Disponível em: <http://www.ascilite.org.au/ajet/ajet24/ostlund. html>. Acesso em: 10 jan. 2015.

PRICHARD, J. S.; STRATFORD, R. J.; BIZO, L. A. Team-skills training enhances collaborative learning. Learning and Instruction, v. 16, n. 3, p. 256265, June 2006. ISSN 0959-4752. Disponível em: $<$ http://www.sciencedirect.com/science/article/pii/ S0959475206000314>. Acesso em: 10 jan. 2015.

RACE, P. 500 [cinq cents] Tips for students. [S. I.]: Basil Blackwell, 1992. ISBN 9780631188513. Disponível em: <http://books.google.com.br/books?id=P6ZhQgAACAAJ>. Acesso em: 10 jan. 2015.

SENÉCAL, C.; KOESTNER, R.; VALLERAND, R. J. Self-regulation and academic procrastination. The Journal of Social Psychology, v. 135, n. 5, p. 607-619, Oct. 1995. ISSN 0022-4545. Disponível em: <http://www. tandfonline.com/doi/abs/10.1080/00224545.1995.97122 34>. Acesso em: 10 jan. 2015.

SOLIMENO, A. et al. The influence of students and teachers characteristics on the efficacy of face-to-face and computer supported collaborative learning. Computers \& Education, v. 51, n. 1, p. 109-128, Aug. 2008. ISSN 0360-1315. Disponível em: <http://dl.acm.org/citation.cfm?id=1371442>. Acesso em: 10 jan. 2015.

TRUEMAN, M.; HARTLEY, J. A comparison between the time-management skills and academic performance of mature and traditional-entry university students. Higher Education, v. 32, n. 2, p. 199-215, Sept. 1996. ISSN 0018-1560. Disponível em: <http://www.jstor.org/ stable/3447923?seq=1\#page_scan_tab_contents>. Acesso em: 10 jan. 2015.

YEDIDIA, M. J. et al. Effect of communications training on medical student performance. JAMA - The Journal of the American Medical Association, v. 290, n. 9, p. 1157-1165, 3 Sept. 2003. ISSN 0098-7484. Disponível em: <http://jama.jamanetwork.com/article.aspx?articleid=197213>. Acesso em: 10 jan. 2015. 